\title{
Codice Romano Carratelli
}

\section{Alessandro Bianchi ${ }^{a}$, Teresa Saeli ${ }^{\mathbf{b}}$}

${ }^{a}$ UniPegaso, Napoli-Roma, Italia, rettore@ unipegaso.it, ${ }^{\mathrm{b}}$ FAI, Vibo Valentia, Italia, ileas@libero.it

\begin{abstract}
The "Romano Carratelli Code" is a late sixteenth century manuscript comprising 99 watercoloured pages (non-contemporary numbering from 1 to 90 in folio), contemporary binding in rigid parchment, handwritten titles on spine, spine with one missing part. It is a splendid fresh watercolour folio depicting the defence system of the coastal areas of Calabria Ultra with illustrations of its garrison towns, castles, watchtowers and surroundings. It includes a detailed description of the various types and features of existing towers and towers under construction as well as an indication of other sites where new watchtowers are necessary. The strategic choice to construct a complete system of watchtowers was a political one made by Charles V and Philip II and the task of drawing up the report was given on the orders of the Viceroy Juan de Zuniga (1586-1595). This document is the most ancient iconography available to historiographers of coastal of Calabria Ultra.
\end{abstract}

Keywords: Fortifications of Calabria - Collection of 99 watercolors - Dated late '500

\section{Introduzione}

Il documento che portiamo all'attenzione di Fortmed 2015 riguarda il sistema di fortificazioni costiere a difesa dell'estrema propaggine del Regno di Napoli: la Calabria Ultra. La realizzazione dell'intero sistema difensivo da contrapporre alle incursioni degli Ottomani era stata avviata da Carlo V fin dall'inizio del suo regno (1520-1556) e poi proseguita da Filippo II (1556-1598). In Calabria il compito venne assunto Juan de Zuniga, Conte di Miranda, durante il periodo in cui fu Vicerè di Napoli (1586-1595), che avviò un imponente lavoro di rilievo e progettazione di un insieme di fortificazioni lungo la costa tirrenica e jonica delle attuali province di Crotone, Catanzaro, Vibo Valentia e Reggio Calabria.

Alla luce delle ricerche attuali, tale lavoro venne svolto in un periodo compreso tra il 1596 e il 1600 e si concluse con la redazione di una raccolta di 99 acquerelli che illustrano con disegni, mappe e annotazioni scritte la situazione esistente e gli interventi da eseguire. E' presumibile che sia stato eseguito da un equipe di militari, topografi e cartografi, ma il nome dell'Autore è ancora sconosciuto. Questo prezioso documento è stato rinvenuto e acquistato alcuni anni fa sul mercato antiquario da un noto bibliofilo di Tropea, l'Avv. Domenico Romano Carratelli, da cui deriva la denominazione di "Codice Romano Carratelli".

\section{La vicenda storica}

Come accennato, la scelta strategica di realizzare un compiuto sistema di torri a difesa delle popolazioni costiere del Regno di Napoli è una scelta politica voluta da Carlo $\mathrm{V}$ e successivamente da Filippo II, che trova attuazione concreta soprattutto durante i governi del Vicerè Pietro di Toledo (1532-1553) e del Vicerè Duca di Alcalà (1559-1575).

Per quanto riguarda la Calabria Ultra, il periodo in cui il programma di fortificazione venne avviato è desumibile dalla comparazione tra i 
dati degli archivi dell'epoca e quelli contenuti nel Codice, in particolare i torrieri e le patenti di nomina, che vengono quasi sempre annotate con la data di concessione.

A tal fine sono di particolare importanza le datazioni di due opere:

- Torre di Capo dell'Armi in territorio di Montebello Jonico, il cui caporale Medio Lopa ha registrato la patente in data $04 / 06 / 1591$;

- Torre Petra Teodosa in territorio di Bova, il cui caporale Minico Jofrida ha registrato la patente in data 25.12.1592.

Di notevole interesse è anche la Torre Tacina nel territorio di Cutro. Essa nel Codice viene indicata come da costruire, mentre il Faglia (Faglia, 1975) annota in base a dati di archivio: "1597 appalto lavori ai mastri fabbricatori per la consegna entro due anni della torre finita"

In conclusione si può ragionevolmente affermare che la stesura del Codice è avvenuta nell'ultimo quinquennio del $1500 \mathrm{e}$, quindi, l'incarico per la sua realizzazione fu quasi certamente una decisione di Juan de Zuniga, Conte di Miranda, Vicerè tra il 1586 e il 1595.

In quel periodo il Regno di Napoli non aveva una cartografia ufficiale e gli stessi lavori ordinati a tal fine ai grandi cartografi del Regno, come Nicola Antonio Stigliola e Mario Cartaro, non venivano diffusi ma anzi erano secretati. $\mathrm{Ne}$ consegue che possiamo ipotizzare che il Codice sia riapparso dopo oltre quattrocento anni, in quanto copia unica e secretata dal Governo Vicereale per motivi di sicurezza.

Da notare che la scoperta del Codice obbliga a retrodatare di circa cento anni le conoscenze che si avevano del territorio calabrese e delle sue rappresentazioni, di cui la più antica fino ad oggi conosciuta era quella tratta dalle incisioni dell'abate Giovan Battista Pacichelli (Pacichelli, 1703).

$\mathrm{Ne}$ consegue che gli studiosi di storia della Calabria antica dovranno riscrivere molte pagine per dare conto del fatto che sono esistiti manufatti di cui si ignorava l'esistenza, che molti di questi erano ubicati in un sito diverso da quello che si presumeva e che alcuni erano di fattezze affatto diverse da quelle che si immaginavano. Dunque anche dal punto di vista storico riteniamo che il Codice rappresenti una scoperta di straordinaria importanza, ed è per questo motivo che nel 2014 la Regione Calabria ha deliberato di candidarlo al Programma UNESCO "Memoria del Mondo" per la salvaguardia del patrimonio documentale.

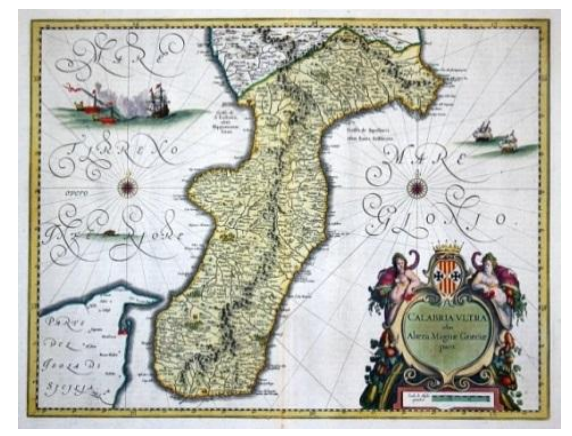

Fig. 1- Calabria Ultra olim Altera Magnae Graeciae pars

\section{La rappresentazione del territorio}

Il Codice è il risultato di un lavoro lungo e difficile condotto in loco con grande professionalità ed assoluta precisione e con riscontri documentali sia per le notizie riportate sia per quanto riguarda la rappresentazione e la toponomastica dei luoghi.

Attraverso la rappresentazione del territorio costiero visto da terra, vengono visualizzate con una descrizione accuratissima le realtà esistenti e le soluzioni possibili al fine di organizzare un efficace sistema difensivo per le città e i territori rivieraschi. Per questo risultano importanti anche le rappresentazioni delle città fortificate e dei castelli prive di note illustrative.

Quanto alle opere fortificate, vengono riportate dettagliatamente le tipologie e le caratteristiche di quelle esistenti e in costruzione e si indicano i luoghi dove si ritiene necessaria la costruzione di nuove fortificazioni, per le quali viene redatto il progetto ed indicate le spese, comprese quelle per i torrieri e i cavallari. In alcuni casi vi sono accenni ai Signori del posto e ai costruttori. 
Alcune immagini tratte dal Codice possono meglio far capire la qualità del documento di cui stiamo parlando.

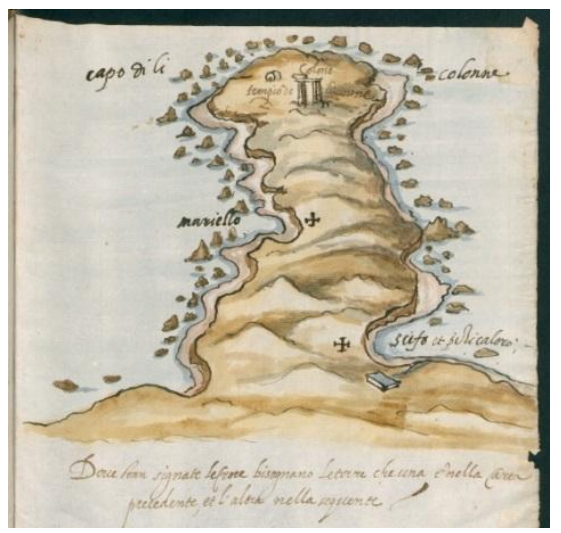

Fig. 1- Capo Colonna

La Fig.1 è relativa a Capo Colonna, una località costiera a poca distanza dalla città di Crotone, nella quale appare una seconda colonna residuata dal Tempio di Hera Lacinia (fine VI sec. a.C.), crollata a causa del terremoto del 1638 e poi scomparsa. Non a caso nel Codice il promontorio è chiamato "Capo delle Colonne".

Da notare che nel medesimo disegno vi sono due croci per le quali la didascalia dice: "Dove son signate le croce bisognano le torre che una è nella carta precedente et l'altra nella seguente".

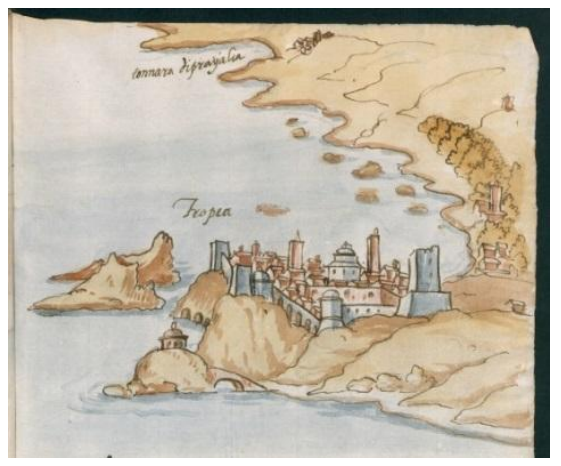

Fig. 2- Tropea

La Fig.2 rappresenta la cittadella fortificata di Tropea, ubicata nel versante tirrenico della provincia di Vibo Valentia. E' rappresentato l'isolotto con l'antica Chiesa di S. Maria dell'Isola, di proprietà dell'Abbazia di
Montecassino. Nella parte alta la scritta indica la "tonnara di prayalia".

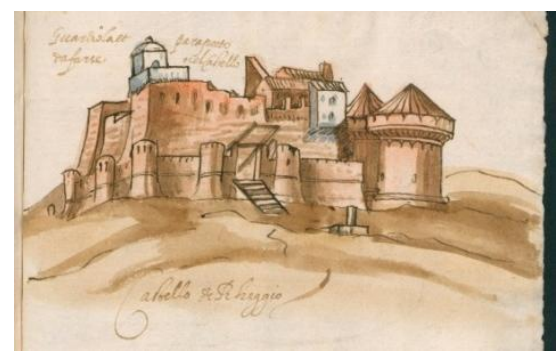

Fig. 3- Castello di Reggio Calabria

La Fig.3 rappresenta il "Castello di Rheggio", noto come "aragonese" ma di origini assai più antiche, con accurati disegni della cinta muraria, dei bastioni, delle torri e della porta d'ingresso. La scritta in alto dice "guardiola et parapetto da farse nel Castello"

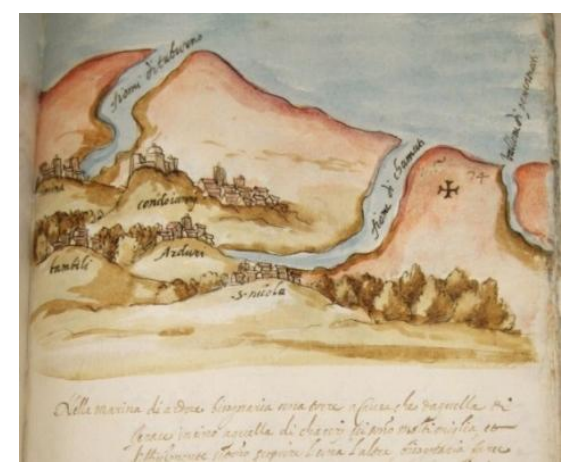

Fig. 4- Marina di Ardore

La Fig. 4 è una splendida rappresentazione del territorio costiero di "Arduri" e dei centri abitati circostanti: "Condoianny, S.Nicola, Bambili, Cimina e delle fiumare che solcano l'entroterra. Nel promontorio sulla destra è disegnata una croce perché "Nella marina di ardore bisognaria una torre aguisa che daquella di Gerace".

In conclusione possiamo affermare che il Codice ha tutte le caratteristiche di un vero e proprio documento di pianificazione del territorio: mostra una visione ampia e di lunga prospettiva del territorio della Calabria Ultra; progetta un insieme di insediamenti difensivi con carattere sistemico, ossia connessi l'uno con l'altro; predispone un piano di spesa per realizzare le opere previste; indica le azioni da eseguire nel 
tempo per l'attuazione del piano. Un atto di pianificazione strategica, che dimostra l'alto livello di capacità di governo in campo militare che caratterizzava all'epoca il vicereame.

\subsection{Una notazione di natura estetica}

Il Codice è anche un'opera di grande valore sul piano estetico, come appare evidente non appena si iniziano a sfogliare le preziose pagine della raccolta che colpiscono, prima ancora per quello che rappresentano, per la bellezza, la fluidità e la trasparenza dei colori delle immagini rese attraverso la difficile tecnica dell'acquerello. Non deve sfuggire che questa qualità estetica è un connotato del tutto casuale, dato che l'opera non nasce certo con tale intento bensì come prodotto di studi di natura militare, presumibilmente affidati ad un gruppo di ingegneri, architetti e topografi. Per fortuna nostra e della cultura mondiale, tra questi si celava un artista che ha conferito ad un prodotto di ingegneria militare un quarto di nobiltà artistica.

\section{La scheda tecnica del manoscritto}

Manoscritto acquerellato cinquecentesco in folio, legatura coeva in pergamena rigida, titoli manoscritti al dorso, una mancanza al dorso. La datazione è stimata alla fine del 1500 , ultimo quinquennio. E' sottoposto a vincolo del Ministero dei Beni ed Attività Culturali con decreto n. 185 dell'8 luglio 2014 emesso dalla Direzione Regionale per i Beni Culturali e Paesaggistici della Calabria. Si tratta di 99 disegni acquerellati di fattura pregevole, a mezza pagina, la gran parte con testo illustrativo in elegante grafia cancelleresca dell'epoca agevolmente leggibile, e per 9 di essi con seguito di scrittura al verso.

La carta si presenta vergata con vergelle molto ravvicinate, impasto omogeneo con pochissimi inclusi al suo interno, i fogli sono filigranati e siglati. I materiali costitutivi, così come la stesura di più strati di collatura, la rendono preziosa e resistente garantendo la tenuta degli acquerelli. Il supporto ha conservato la sua planarità nonostante l'apporto di acqua adoperata per l'acquerello e non appare avere comportamenti diversi fra la zona dipinta e l'area interessata dall'inchiostro. Quest'ultimo, ferro-gallo-tannico, di color seppia con le caratteristiche storicizzate degli inchiostri in uso nella seconda metà del Cinquecento, ha mantenuto un'ottima stabilità senza evidenti manifestazioni di alterazioni a dimostrazione della sapiente formulazione dei suoi ingredienti costitutivi. Certamente anche l'ambiente di conservazione è stato ottimale per le condizioni nelle quali ci è pervenuto il manoscritto con indici termo idrometrici più o meno costanti.

Gli acquerelli sono stati eseguiti con visuale da terra a mare, da un esperto conoscitore della tecnica, che sorprende anche per la modernità della esecuzione. Il manufatto cartaceo è stato realizzato perché potesse durare nel tempo, vi ricorrono diversi motivi di filigrana - l'angelo e la balestra in modo particolare - con l'apposizione di iniziali, probabilmente ad indicare il cartaro.

Lo stato di conservazione è ottimo, anche se va segnalato un naturale ingiallimento $\mathrm{e}$ un grossolano restauro ottocentesco nel terzo gruppo di carte, dove il terzo e quarto foglio presentano interventi di restauro conservativo non proprio fedeli alle reali mancanze, con intervento anche sui bordi e senza rispettare la geometria della vergatura.

In conclusione si tratta di uno splendido manoscritto cartaceo acquerellato di straordinaria freschezza di fine '500, che rappresenta ed illustra il problema della difesa della zona costiera di Calabria Ultra attraverso la raffigurazione delle sue città fortificate, dei suoi castelli, delle sue torri e del suo territorio.

\section{References}

Atlas Maior, (1667). Calabria Ultra olim Altera Magnae Graeciae pars, Blaeu. Ed. Amsterdam.

Faglia, V. (1975). Tipologia delle Torri Costiere nel Regno di Napoli, Roma, vol. II, p. 321

Pacichelli, G.B. (1997). Il Regno di Napoli in prospettiva, Forni Editore, Sala Bolognese (rist. anast., Ed. orig. 1703, Napoli). 